\title{
Reflets
}

Revue ontaroise d'intervention sociale et communautaire

\section{Ils ne peuvent pas enseigner dans nos écoles : le dilemme des étudiants-maîtres des minorités visibles nés au Canada}

\section{Donatille Mujawamariya}

Volume 6, numéro 2, automne 2000

Problèmes sociaux en Ontario français

URI : https://id.erudit.org/iderudit/026319ar

DOI : https://doi.org/10.7202/026319ar

Aller au sommaire du numéro

Éditeur(s)

Reflets : Revue ontaroise d'intervention sociale et communautaire

ISSN

1203-4576 (imprimé)

1712-8498 (numérique)

Découvrir la revue

Citer cet article

Mujawamariya, D. (2000). Ils ne peuvent pas enseigner dans nos écoles : le dilemme des étudiants-maîtres des minorités visibles nés au Canada. Reflets, 6(2), 138-165. https://doi.org/10.7202/026319ar

Tous droits réservés (C) Reflets : Revue ontaroise d'intervention sociale et communautaire, 2000
Ce document est protégé par la loi sur le droit d'auteur. L'utilisation des services d'Érudit (y compris la reproduction) est assujettie à sa politique d'utilisation que vous pouvez consulter en ligne.

https://apropos.erudit.org/fr/usagers/politique-dutilisation/ 


\title{
Ils ne peuvent pas enseigner dans nos écoles $\square$ : le dilemme des étudiants-maîtres ${ }^{1}$ des minorités visibles ${ }^{2}$ nés au Canada
}

\author{
Donatille Mujawamariya \\ Professeure, Faculté d'éducation ${ }^{3}$, Université d'Ottawa et chercheure \\ associée au Centre de Recherche Interuniversitaire sur la Formation et la \\ profession enseignante - CRIFPE.
}

Bien qu'on semble agréer en parole au besoin d'assurer l'égalité dans une société pluraliste, en réalité, les particuliers et les institutions sont beaucoup plus déterminés à maintenir le statu quo afin de stabiliser leur puissance ou même de l'augmenter (Henry et Tator 1994 : 1).

Le contenu des programmes d'enseignement et les attitudes des professeurs et des enseignants face aux élèves des autres communautés ethniques et culturelles dans les universités et les écoles secondaires et primaires ont fait l'objet de nombreuses critiques. Plusieurs rapports et études ont fait état du racisme dans les institutions d'enseignement (Alladin 1996; Axelrod 1997; Dei et al. 1997; Friesen 1993; Gollnick et Chinn 1998; Henry et al. 2000; Martin et Warburton 1998; Palmer 1984; Satzewich 1998; Stanley 1995). Ceux-ci mettent en évidence l'eurocentrisme du curriculum, qui ne présente qu'une culture et qui fait rarement allusion aux questions d'ethnie et de discrimination. En outre, les enseignants appartenant à la culture majoritaire véhiculent parfois certains préjugés à l'égard d'autres cultures (Barton, Watkins et Jarjoura 1997; Beckham 1988; Dei et al. 1997; Huntley 1993; 
«...bien que le nombre d'immigrants provenant des minorités visibles et de groupes ethniques soit en hausse au Canada... leur représentation parmi le corps enseignant et à la formation des maîtres demeure très limité."
Martin et Warburton 1998; McConnell Castle 1993; Mujawamariya, sous presse; Nixon-Ponder 1998; Watt et Roessingh 1994). Selon ces auteurs, les élèves issus des cultures minoritaires peuvent plus difficilement contribuer à la construction du savoir en classe. Ils ont également plus de difficulté à donner leur opinion et reçoivent moins souvent de commentaires constructifs. À cause de ces contraintes, ils sont parfois mal évalués et leurs travaux mal notés. Ils sont parfois victimes du mauvais traitement d'autres élèves et ils peuvent faire l'objet d'intimidation de la part de leurs enseignants. De façon générale, ils sont plus susceptibles de vivre des situations d'échec scolaire et de décrochage. Quant aux administrateurs dans les milieux d'éducation, ils semblent avoir tendance à nier ces phénomènes (Clay, Gadhia et Wilkins 1991; Dei 1993; Goodwin et Nacht 1983; Grant 1993; Martin et Warburton 1998; Sleeter 1993; Zeichner et Hoeft 1996).

Par ailleurs, bien que le nombre d'immigrants provenant des minorités visibles et de groupes ethniques soit en hausse au Canada (Statistique Canada 1998), leur représentation parmi le corps enseignant et à la formation des maitres demeure très limité. En effet, pendant que la population canadienne de souche vieillit et que son taux de natalité décroît, sa diversité ethnique et culturelle augmente. Selon Dei et al. (1997 : 9), la population canadienne de souche a connu un taux de croissance de $9 \%$ de 1986 à 1991 alors que, pour cette même période, le taux de croissance de la population des minorités visibles est passé à $58 \%$. En Ontario, entre 1991 à 1996, la population totale est restée la même, mais celle des minorités visibles a connu une croissance de $3 \%$ (Dei 1997; Statistique Canada 1993, 1998). En 1996, la ville de Toronto abritait 79,5\% des minorités visibles de la province. Selon ces mêmes données, au moins $72,5 \%$ des minorités visibles au Canada habitent dans les trois villes de Toronto (41,8\%), Vancouver $(17,6 \%)$ et Montréal (13,2\%) (Statistique Canada 1998). Le reste de cette population se répartit dans les autres villes selon les proportions suivantes : Calgary, 4,0\%, Ottawa-Hull, 3,6 \% et Edmonton, 3,6 \%. Ces données indiquent que les élèves, surtout ceux des écoles urbaines, proviennent de cultures, d'ethnies et de langues de plus en plus diverses. Néanmoins, il y a peu d'en- 
"...il peut exister des barrières systémiques qui nuisent à l'entrée des membres des minorités visibles dans la profession enseignante de même qu'à leur admission et à leur réussite au sein $d u$ programme de formation initiale.» seignants provenant de groupes minoritaires auxquels les élèves peuvent s'identifier (Birch et Elliot 1993; Carr 1995; Cheng et Soudack 1994; Dei 1996; Henry et al. 2000; Huntley 1993; MEO 1994; Martin et Warburton 1998; Programme d'équité d'emploi et d'éducation 1996; Smith 1989; Solomon 1997, 1999; TalbertJohnson et Tillman 1999). Dans une étude récente, Herry (1995 : 3) souligne qu' "en Ontario, le personnel enseignant des écoles primaires et secondaires ne compte qu'un pour cent (1\%) de membres de minorités raciales, de groupes ethnoculturels ou des Premières Nations alors qu'ils représentent environ seize pourcent $(16 \%)$ de la population scolaire ".

À ce propos, le ministère de l'Éducation et de la formation de l'Ontario (1994) adhère à l'idée qu'il peut exister des barrières systémiques qui nuisent à l'entrée des membres des minorités visibles dans la profession enseignante de même qu'à leur admission et à leur réussite au sein du programme de formation initiale. Notre étude vise à identifier ces barrières et les solutions plausibles à y apporter, en tenant compte des perceptions des enseignantsassociés sur la pratique enseignante des étudiants-maitres des minorités visibles. Le texte qui suit comprend quatre sections. La première traite du but et des objectifs poursuivis dans cette recherche, tout en précisant le cadre théorique qui la sous-tend. La deuxième section précise les aspects méthodologiques qui ont guidé la cueillette et l'analyse des données alors que la troisième porte sur la présentation et la discussion des résultats. Enfin, la conclusion souligne les apports de cette étude et identifie les pistes de recherches subséquentes sur le sujet.

\section{But et objectifs de l'étude}

Ce projet est né d'un certain constat à propos des difficultés vécues par les étudiants membres des minorités visibles inscrits au programme de formation à l'enseignement de l'Université d'Ottawa. Ces difficultés semblent s'accentuer au moment des stages de pratique à l'enseignement, une composante majeure de 
"...plusieurs facteurs découragent la poursuite d'études universitaires chez les personnes de minorités visibles. Ces facteurs touchent leur statut minoritaire, la réaction des institutions à leur égard, les problèmes d'ajustement et les exigences de performance scolaire." la formation en vue de l'obtention d'une carte de compétence (brevet d'enseignement) de l'Ontario.

Selon Beckham (1988), plusieurs facteurs découragent la poursuite d'études universitaires chez les personnes de minorités visibles. Ces facteurs touchent leur statut minoritaire, la réaction des institutions à leur égard, les problèmes d'ajustement et les exigences de performance scolaire. C'est pourquoi, comme le souligne Beckham, le problème de l'intégration persiste encore à ce jour. Toutefois, au Canada, la plupart des recherches sur l'intégration des étudiants de minorités visibles et ethnoculturelles se sont adressées à des programmes différents de celui de la formation à l'enseignement ( Martin et Warburton 1998; Young 1994), à des populations d'expression anglaise (Carr 1995; Solomon 1997,1999) ou à des enseignants et élèves dans les écoles (Cazabon et al. 1998; Dei et al. 1997; Langlois 1998). Les travaux de Salomon, qui analysent les perceptions et les attitudes d'enseignants-associés anglophones face aux étudiants-maitres de race blanche et noire jumelés au même enseignant, concluent à la marginalisation et au mauvais traitement que ces enseignantsassociés réservent aux étudiants-maitres de race noire. Or, en Ontario, il est connu que les Franco-Ontariens sont défavorisés par rapport aux anglophones et qu'ils sont constamment préoccupés par des questions touchant : à la compréhension de leurs besoins et opinions par un gouvernement anglophone; à la fierté de pouvoir jouir pleinement de leur identité francophone; à la justice pour accéder aux services essentiels répondant à leurs besoins; aux institutions dans lesquelles ils voudraient participer pleinement et à leurs droits aux services en français. Il va de soi qu'à côté des Franco-Ontariens, les minorités visibles et ethnoculturelles d'expression française sont doublement défavorisées (Office des affaires francophones 1991). Cependant, cela ne nous permet pas de présumer de la nature des rapports entre enseignants-associés et étudiants-maîtres de minorités visibles et ethnoculturelles.

Sans être une étude sur l'histoire des Franco-Ontariens, notre recherche se situe dans le processus de compréhension de la lutte qu'ils mènent pour leurs droits. Et comme le disait si bien Wade 
$(1974: 79)$ :

"Cette étude s'intéresse plus particulièrement au contexte de formation de futurs enseignants membres des minorités visibles, lequel semble un terrain de recherche quasi vierge, en dépit de l'abondante littérature sur l'encadrement des stagiaires en général..."
L'histoire des Franco-Ontariens n'est pas seulement une question de "nous " francophones contre les " autres " anglophones. En effet, les autres sont bien divers et divisés entre eux; ils comprennent non seulement les Irlandais, mais aussi les Écossais, les Anglais, les Américains et les Nouveaux Canadiens. Il faut distinguer les attitudes de chacun de ces groupes envers les Franco-Ontariens, et viceversa.

C'est dans cette optique que nous nous préoccupons, selon l'expression de Wade, de «l'action réciproque » entre les Franco-Ontariens et les Nouveaux Canadiens dont la langue d'usage est le français. Cette étude s'intéresse plus particulièrement au contexte de formation de futurs enseignants membres des minorités visibles, lequel semble un terrain de recherche quasi vierge, en dépit de l'abondante littérature sur l'encadrement des stagiaires en général (Applegate et Lasley 1982; Biron et al. 1995; Boivert 1987; Bujold et Côté 1996; Descôteaux 1992; Lavoie et Garant 1995; Locke 1984; Paré 1993; Spallanzani et al. 1992;Villemure 1989; Zeichner 1980).

Notre étude s'inscrit dans le prolongement des initiatives entreprises par la Faculté d'éducation de l'Université d'Ottawa, par le biais d'un programme pilote d'initiatives antiracistes s'étant déroulé pendant l'année universitaire 1994-1995 (Herry 1995). Celui-ci avait pour but d'adapter le programme de formation initiale en français afin qu'il réponde adéquatement aux besoins des membres des minorités visibles, des groupes ethnoculturels ${ }^{4}$ et des Premières Nations ${ }^{5}$. Le point innovateur de la présente recherche réside dans le fait qu'elle propose une étude systématique des difficultés d'intégration auxquelles font face les étudiantsmaîtres des minorités visibles. Elle vise, d'une part, à analyser les perceptions des enseignants-associés au regard de la pratique enseignante des étudiants-maîtres des minorités visibles et d'autre part, à identifier des solutions possibles pour faciliter l'adaptation de cette clientèle aux milieux de stage et aux institutions d'enseignement en Ontario français. 


\section{Méthodologie}

"Il s'agit d'une recherche-action d'inspiration constructiviste qui nous permettra de recueillir les opinions des principaux intervenants sur les différents aspects de la formation des enseignants, en particulier sur l'encadrement des stagiaires en milieu scolaire."
L'état quasi embryonnaire de la recherche sur les formateurs des étudiants des minorités visibles en formation des enseignants au Canada, (Wideen et al. 1998; Ouellet 1999) et en particulier en Ontario français, nous impose une étude exploratoire, c'est-àdire "souple, dont le but est d'obtenir une compréhension préliminaire et descriptive d'une situation en vue de préciser une première problématique, d'émettre intuitivement des hypothèses et d'évaluer la pertinence d'études ultérieures plus systématiques " (Legendre 1988 : 485). Il s'agit d'une recherche-action d'inspiration constructiviste qui nous permettra de recueillir les opinions des principaux intervenants sur les différents aspects de la formation des enseignants, en particulier sur l'encadrement des stagiaires en milieu scolaire. C'est pourquoi nous recourons à une approche qualitative de type thématique dans laquelle les participants sont conviés à nous parler de leurs expériences en tant qu'enseignants-associés d'étudiants-maitres de minorités visibles. Nous avons privilégié des entrevues de type semi-structuré afin de permettre à l'interlocuteur d'élaborer ses réponses à sa guise; notre rôle consistait à lui suggérer des thèmes généraux ayant trait, directement ou indirectement, à la pratique enseignante des stagiaires de minorités visibles.

Treize enseignants-associés, huit femmes et cinq hommes, tous recrutés sur une base volontaire, ont pris part à cette étude. Ces derniers encadraient 14 étudiants des minorités visibles inscrits aux programme de formation à l'enseignement à l'Université d'Ottawa au cours de la session d'hiver 1998. Deux étaient inscrits aux cycles intermédiaire-supérieur, un aux cycles moyen-intermédiaire et onze aux cycles primaire-moyen. Les 13 enseignantsassociés relèvent de dix écoles (cinq catholiques, cinq publiques) appartenant à quatre conseils scolaires ontariens. Il convient de mentionner que ces enseignants-associés étaient tous des FrancoOntariens "de souche" de race blanche, à l'exception d'un enseignant émigré d'Europe depuis quelques années. Par ailleurs, parmi les 14 étudiants que ces enseignants-associés encadraient, 
"Le questionnaire d'entrevue a été préparé de façon à rendre compte de la plupart des aspects susceptibles d'influer directement ou indirectement sur l'intégration des minorités visibles dans la profession enseignante,..." quatre sont nés au Canada, cinq sont arrivés au Canada avant l'âge de 7 ans, un est au Canada depuis plus de 30 ans et les quatre autres sont installés au Canada depuis moins de 7 ans.

Le questionnaire d'entrevue a été préparé de façon à rendre compte de la plupart des aspects susceptibles d'influer directement ou indirectement sur l'intégration des minorités visibles dans la profession enseignante, selon les perceptions qu'entretiennent les enseignants-associés sur la pratique enseignante des candidats des minorités visibles. L'entrevue portait entre autres sur la connaissance de la culture de l'étudiant-maitre qu'avait son enseignantassocié, sur le contact avec cet étudiant-maitre, la qualité de son intervention pédagogique, l'évaluation de sa pratique enseignante, son comportement professionnel et ses qualités personnelles ainsi que sur des suggestions pour aider les étudiants de minorités visibles à contrer les difficultés rencontrées en milieu de stage.

Notre étude prenant son origine à l'université, il a fallu tout d'abord sensibiliser les étudiants-maîtres des minorités visibles à celle-ci et les inviter à y participer ${ }^{6}$. Par la suite, les directeurs et les directrices des écoles associées ont été contactés par téléphone, pour être informés du projet. Ils ont alors reçu un formulaire de consentement autorisant la conduite des entrevues auprès des enseignants-associés qui superviseraient les étudiants participant à la recherche. Les enseignants-associés ont été rencontrés individuellement afin de recevoir des explications sur le but et les implications de la recherche. Les entrevues individuelles de type semi-structuré dont la durée variait entre 30 et 45 minutes ont été enregistrées sur bande audio et se sont déroulées en mars et avril 1998.

Nous nous sommes inspirée de modèles de Giorgi (1985) et de L'Écuyer (1987) pour l'analyse des verbatims de ces entrevues. Pour chaque entrevue, ce travail d'analyse comprenait : un minimum de trois lectures pour se familiariser avec le verbatim; une division du texte en unités de sens; une transcription épurée en enlevant les détails inutiles; une expression du sens contenu dans chaque unité de sens; une clarification par thème; une expression $\mathrm{du}$ sens global par thème et enfin une expression du sens global des divers thèmes. C'est ce qui nous a permis de regrouper les 
opinions des enseignants-associés interviewés dans les catégories émergentes dont il est question dans la section suivante.

Pour des raisons de confidentialité, les noms des enseignantsassociés ont été remplacés par un code EA [pour EnseignantAssocié] suivi chaque fois d'un chiffre de 1 à 13 , pour les 13 enseignants-associés. Après chaque citation, nous précisons également le statut de l'étudiant-maitre encadré par l'enseignant en question : EM [pour Étudiant-Maitre] né au Canada, EM a grandi au Canada, EM au Canada depuis quelques années ou EM récent au Canada. La pertinence de préciser le statut de l'étudiant-maître se justifie de manière explicite dans l'interprétation que nous faisons des propos tenus par les enseignantsassociés respectifs.

\section{Les enseignants-associés face à la diversité culturelle des étudiants-maîtres}

«La présente étude permet,... d'appréhender comment se manifeste la culture dominante francoontarienne, dans les expériences et les perceptions d'acteurs qui oeuvrent au sein $d u$ système d'enseignement franco-ontarien. "
Dans sa politique du multiculturalisme énoncée dans la Loi sur le multiculturalisme canadien de 1988, le gouvernement reconnait la diversité et s'engage à préserver et à mettre en valeur l'héritage multiculturel des Canadiens. Cependant, de nombreux auteurs critiques vis-à-vis du multiculturalisme (Henry et al. 1995; Young 1990) indiquent qu'au lieu de reconnaître et de refléter les différences ethniques et culturelles, la politique officielle de multiculturalisme encourage les notions contradictoires que sont l'assimilation, la gestion et la prise en compte des différences au sein d'une majorité ethnocentrique et homogène. La communauté franco-ontarienne dite "de souche ", bien que victime de son identité francophone par rapport à la majorité anglophone, ne semble pas échapper à ce phénomène vis-à-vis des minorités visibles francophones de l'Ontario. La présente étude permet, si peu soit-il, d'appréhender comment se manifeste la culture dominante francoontarienne, dans les expériences et les perceptions d'acteurs qui oeuvrent au sein du système d'enseignement franco-ontarien. 


\section{Le rejet de la différence}

"...il ressort de leurs discours des éléments qui témoignent d'un certain malaise, d'une certaine méfiance face à l'expérience qu'ils vont vivere, d'un sentiment de peur face à l'inconnu. "
"Comment décririez-vous votre premier contact avec les étudiants-maitres de minorités visibles? » était l'une des questions d'entrevue adressées aux enseignants-associés. La majorité des enseignants-associés rapportent avoir eu avec les étudiants-maitres des minorités visibles un premier contact positif, excellent ou aussi bien qu'avec n'importe quel autre étudiant. Par contre, il ressort de leurs discours des éléments qui témoignent d'un certain malaise, d'une certaine méfiance face à l'expérience qu'ils vont vivre, d'un sentiment de peur face à l'inconnu. Certains d'entre eux avouent même avoir anticipé qu'il y aurait des problèmes juste à la vue du nom du stagiaire, lequel nom ne semblait pas habituel aux yeux de l'enseignant-associé.

"Moi j'ai eu peur quand j'ai vu son nom tout simplement sur le petit document qui disait que j'avais une stagiaire, j'ai eu un peu de misère à lire son nom et puis je me suis dit: bon quelle nationalité est-ce que c'est? Je me suis dit : "Bon j'espère que je n'aurai pas de problème au niveau langage" mais je n'en ai pas eu du tout" (EA4, son EM a grandi au Canada).

"Quand j'ai vu le nom, j'ai tout de suite su que c'était une haitienne ou probablement une africaine. J'étais un peu craintive, mais ça n'a pas pris de temps qu'on s'est senti à l'aise et puis qu'on a bien communiqué et puis mes craintes se sont dissipées très rapidement. J'étais craintive mais je voulais l'accueillir puis la sentir bienvenue dans ma classe et puis que les élèves aussi l'accueillent parce que dans notre école on n'a pas beaucoup de minorités visibles " (EA6, son EM a grandi au Canada).

"La dame s'est présentée le vendredi précédant la semaine de stage et elle a pu avoir un contact avec les jeunes et j'ai bien aimé la façon qu'elle a présenté son 
"Certains enseignantsassociés ne se sont pas gênés de déclarer que l'accent pouvait représenter une barrière à la poursuite de la carrière enseignante de ces minorités visibles. " nom parce qu'il est complètement différent du nôtre " (EA3, son EM est récent au Canada).

Huit des 13 enseignants-associés interviewés semblent avoir été agacés par l'accent de l'étudiant-maître encadré. Même lorsque les autres aspects de la pratique enseignante s'avéraient réussis, l'accent ne pouvait passer inaperçu, histoire de souligner encore une fois que l'étudiant est " autre ». Certains enseignants-associés ne se sont pas gênés de déclarer que l'accent pouvait représenter une barrière à la poursuite de la carrière enseignante de ces minorités visibles.

"Une chose que j'ai notée, c'est l'accent 》(EA12, son EM est récent au Canada).

"Elle a un léger accent et je trouve que c'est spécifique et c'est plus remarquable.

Mes élèves doivent se faire à son vocabulaire et à sa tonalité plus grave qu'à moi » (EA4, son EM a grandi au Canada).

"Si elle devait enseigner dans la région, elle devra être consciente que son accent pose un problème " (EA6, son EM est récent au Canada).

Quand le nom n'est pas source de suspicion et que l'étudiant des minorités visibles de deuxième ou troisième génération a pris l'accent local, c'est plutôt son apparence physique qui est en jeu. C'est ainsi que cette étudiante que nous avons surnommée Julie a dû fournir des explications sur son identité à son enseignantassocié : ses ancêtres sont d'origine asiatique.

"Au début, le contact a été par téléphone. Quand je l'ai entendue [poursuit l'enseignant-associé], je n'ai pas remarqué l'accent du tout puisqu'elle s'est présentée comme Julie. Puis sur la fenille, le nom que j'avais ce n'était pas du tout Julie. Elle [l'étudiante-maître] a dit : "mon nom canadien c'est Julie et mon nom [authentique] c'est le nom que tu as sur la feuille” " (EA13, son EM est né au Canada). 
"...dans leurs discours, les enseignants-associés ont plutôt tendance à généraliser, ce qui nous laisse croire que leurs jugements sont, dans une certaine mesure, teintés de stéréotypes véhiculés sur les minorités visibles dans la société. "
Relativement à la question qui portait sur la connaissance qu'avait l'enseignant-associé de la culture de l'étudiant-maitre et sur l'importance de celle-ci dans la relation d'aide auprès du stagiaire, 12 des 13 enseignants-associés confirment qu'il est important de connaitre la culture des étudiants de minorités visibles. Cependant, bien que huit des 13 enseignants-associés interviewés en étaient à leur première expérience d'encadrement de minorités visibles et que trois parmi eux avouent qu'ils ne connaissaient pas la culture de leurs étudiants-maîtres, 12 enseignants-associés soutiennent que la culture des étudiants-maitres des minorités visibles est à l'origine des difficultés que connaissent ces stagiaires. N'y a-t-il pas là une indication claire que, dans leurs discours, les enseignants-associés ont plutôt tendance à généraliser, ce qui nous laisse croire que leurs jugements sont, dans une certaine mesure, teintés de stéréotypes véhiculés sur les minorités visibles dans la société.

"Spécialement pour le type qu'il est, qui est de race noire, son approche est totalement différente et puis ça lui cause des problèmes de discipline " (EA3, son EM est récent au Canada).

"La gérance de temps, il ne l'a pas; comme mon autre dame ne l'avait pas. Pour eux, le temps c'est comme si ça n'existait pas " (EA5, son EM a grandi au Canada).

"L'EM a besoin de travailler son français " (EA8, son EM est né au Canada).

"Les EM des minorités visibles en général n'ont pas la facilité avec l'anglais; ils ont des voix très douces ou une tonalité faible " (EA6, son EM est récent au Canada).

"L'EM avait une faible connaissance de la matière, j'aimerais qu'on m'envoie de la minorité qui a réussi à l'Université avec 90\% " (EA5, son EM a grandi au Canada). 
"Je remarque que l'ensemble des stagiaires que j'ai eus qui sont des minorités visibles, pour eux la discipline en salle de classe, la vision globale de la classe semblent être quelque chose moins important. Dû̀ à leur culture, ce sont des gens très passifs non préoccupés du degré d'indiscipline et de mouvements autour d'eux; ils peuvent vivre dans cette atmosphère-là " (EA5, son EM a grandi au Canada).

"Les difficultés sont constantes parmi les minorités visibles" (EA1, son EM est né au Canada).

Des études antérieures sur l'encadrement des stagiaires (Bujold et Côté 1996) révèlent que l'intervention pédagogique, la gestion de classe (discipline), la connaissance des objectifs et des programmes et la gestion du temps viennent en tête des principaux problèmes (au nombre de 16) que rencontrent les stagiaires en général et ne sont pas uniquement le lot des étudiants-maitres de minorités visibles tel que le prétendent ces enseignants-associés. Qu'on le veuille ou non, ces stéréotypes sont des stimulants d'iniquité conduisant les enseignants-associés à avoir des attentes tantôt faibles, tantôt élevées, selon qu'il s'agisse de l'apprentissage ou de l'évaluation. Parmi les difficultés, ils mentionnent celles relatives à la discipline, à la planification et à l'organisation des cours, à la maîtrise de la matière enseignée, sur les plans de l'expression verbale et de l'implication dans la vie de l'école.

"...la plupart des enseignants-associés que nous avons interviewés ne semblent pas préoccupés d'aider le stagiaire à développer un style professionnel, mais semblent plutôt passer à la loupe ses erreurs, les comparent à ses homologues « de souche »." Pourtant, cette liste est parfaitement compatible avec celle dressée par Bujold et Côté (1996: 31) selon les perceptions de 236 intervenants, majoritairement des enseignants du primaire et du secondaire ayant encadré des stagiaires.

Par ailleurs, dans leur processus d'accompagnement auprès des stagiaires des minorités visibles, la plupart des enseignants-associés que nous avons interviewés ne semblent pas préoccupés d'aider le stagiaire à développer un style professionnel, mais semblent plutôt passer à la loupe ses erreurs, les comparent à ses homologues "de souche ".Vraisemblablement, ces stagiaires font cavalier seul et leur pratique est jugée inadaptée par rapport à la pratique de référence animée d'une mission, non-avouée, d'assimilation à la 
" "Son origine affecte sa vision de l'enseignement dans le sens qu'elle a tendance à être plus magistrale que manipulatrice (interactive)..."

« "Comparativement aux Canadiens de souche, les minorités s'impliquent moins dans la vie scolaire, ils sont moins autonomes "..." norme enkystée. L'iniquité systémique laissant ainsi voir des patrons clairs, les minorités visibles seraient des groupes à habiletés et à potentiel restreints.

"Le fait qu'elles sont d'une autre race,je les ai trouvées un petit peu plus traditionnelles dans leur approche d'enseignement... moins portées à faire des activités décloisonnées. J'ai trouvé que mes franco-ontariennes que j'ai eues dans les quatre, cinq ans avaient des techniques plus dernière mode " (EA6, son EM est récemment arrivé au Canada).

"Son origine affecte sa vision de l'enseignement dans le sens qu'elle a tendance à être plus magistrale que manipulatrice (interactive), le choix de ses appuis visuels était beaucoup plus magistral que d'autres étudiantsmaîtres que j'ai eus " (EA10, son EM est récent au Canada).

"Elle a été élevée avec le système français. Je la vois interagir avec les enfants puis elle se trouve à être ferme comme dans le système français; elle a besoin de s'ajuster aux exigences franco-ontariennes, à savoir que les attentes du système français et du système francoontarien different " (EA10, son EM est récemment arrivé au Canada).

"Comparativement aux Canadiens de souche, les minorités s'impliquent moins dans la vie scolaire, ils sont moins autonomes » (EA3, son EM est récemment arrivé au Canada).

"L'EM ne pouvait pas s'adapter à notre enseignement, à notre façon de faire les choses " (EA1, son EM est né au Canada).

Cette lacune attribuée ici aux étudiants-maîtres des minorités visibles est identifiée dans l'étude de Bujold et Côté (1996 : 33) comme étant l'un des principaux problèmes que rencontrent les enseignants-associés en supervision de stage. En effet, la relation 
"On ne peut donc pas dire que les enseignantsassociés qui présentent des lacunes dans la relation d'aide agissent comme de véritables mentors, parrains ou guides auprès des stagiaires de minorités visibles pendant qu'ils passent leur temps à les mettre à l'épreuve, vraisemblablement à cause de leurs différences. Ils se comportent plutôt comme des agents de contrôle. " d'aide occupe le troisième rang dans une liste de 14 problèmes énumérés. Paré (1993) fait un rapprochement entre la relation d'aide et la disponibilité (présence) de l'enseignant-associé dans l'encadrement du stagiaire et associe à cette disponibilité une signification profonde entre les deux partenaires qui cherchent à évoluer ensemble. Sans cette présence, il semble qu'il soit pratiquement impossible d'atteindre le niveau d'évolution le plus élevé dans la relation d'aidant, celui du devenir, de la croissance personnelle (Paré 1993; Bujold et Côté 1996). On ne peut donc pas dire que les enseignants-associés qui présentent des lacunes dans la relation d'aide agissent comme de véritables mentors, parrains ou guides auprès des stagiaires de minorités visibles pendant qu'ils passent leur temps à les mettre à l'épreuve, vraisemblablement à cause de leurs différences. Ils se comportent plutôt comme des agents de contrôle.

Au Canada, la question " d'où viens-tu, originairement? " est passée dans les moeurs des gens pour vous manifester que l'on ne vous reconnait pas des siens! Cette question émane de votre accent, de la couleur de votre peau ou de votre nom (Mujawamariya 1998). Or, le Canada étant un pays multiculturel, il nous semble paradoxal de vouloir reconnaitre un Canadien par son nom ou par son accent et encore moins par la couleur de sa peau. Malheureusement, la plupart des enseignants-associés interviewés font la distinction parmi les francophones de l'Ontario sur la base de leur nom, de leur accent et de la couleur de leur peau. Ainsi les minorités visibles francophones passent difficilement pour des Canadiens et leur premier contact a souvent provoqué de la crainte chez les enseignants-associés. En effet, dans leurs discours, les enseignantsassociés semblent traiter d'étrangers les étudiants-maîtres qu'ils encadrent alors que ces derniers sont tous de nationalité canadienne et francophones au même titre que ces enseignants-associés. Ces expériences de stage vécues par les étudiants-maitres des minorités visibles et décrites par les enseignants-associés participant à cette étude semblent contribuer au renforcement de la marginalisation des minorités visibles francophones en les tenant à l'écart de la profession enseignante à cause de l'accent, de la couleur de la peau ou tout simplement à cause du nom. 


\section{Le manque d'objectivité au nom de l'égalité}

"...les enseignantsassociés ont tendance, pour «le principe d'équité ", à uniformiser leur attitude et à ne pas tenir compte de la diversité. "

"Je pense que les enseignants doivent être tous pareils, avoir tous les mêmes critères d'évaluation et rentrer dans le même cadre "..."
Dans les propos cités ci-après, les enseignants-associés reconnaissent que la culture influe sur la vision que l'on se fait de l'enseignement. Par ailleurs, il ne semble pas y avoir de doute qu'il y ait plus que l'habileté académique et le potentiel individuel dans les décisions scolaires prises par et pour l'étudiant-maître (Varpalotai 1995). Cependant, les enseignants-associés ont tendance, pour « le principe d'équité ", à uniformiser leur attitude et à ne pas tenir compte de la diversité. C'est ce qui explique qu'aucun de ces enseignants ne ressent le besoin de mieux adapter le formulaire d'évaluation à ces étudiants, malgré le poids qu'ils semblent accorder à la culture dans l'enseignement. Les opinions qu'ils tiennent à ce sujet semblent confirmer cette assertion.

"Je crois que l'acte d'enseigner est le même, les curriculum sont les mêmes, la capacité de savoir s'adapter à l'élève ou de bien questionner est la même... moi je crois que l'évaluation devrait rester la même " (EA6, son EM est récent au Canada).

"Je ne serais pas d'accord pour l'adapter [formulaire d'évaluation] pour eux parce que ça fait quelque chose de spécial pour eux alors qu'ils sont des étudiants comme les autres. Je veux qu'ils aient les mêmes compétences, les mêmes critères d'évaluation. Je pense que les enseignants doivent être tous pareils, avoir tous les mêmes critères d'évaluation et rentrer dans le même cadre " (EA4, son EM a grandi au Canada).

"Je pense qu'ils devraient être évalués comme tout autre élève [étudiant-maître]s'ils veulent enseigner dans notre système. Je pense qu'il faut avoir les mêmes critères peu importe l'étudiant qu'on reçoit; je trouve que c'est juste » (EA13, son EM est né au Canada). 
C'est d'ailleurs le même discours qu'ils tiennent face à la connaissance de la culture de l'étudiant-maitre. Pour certains, cette connaissance est importante car elle permet de comprendre et d'encadrer adéquatement l'étudiant-maitre, alors que pour d'autres il n'y a pas d'intérêt à connaitre cette culture car leur préoccupation est de former de bons enseignants peu importe leur culture ou

"...ils prônent l'égalité dans le traitement tout en renforçant, consciemment ou non, les inégalités. " leur couleur : ils prônent l'égalité dans le traitement tout en renforçant, consciemment ou non, les inégalités. Paradoxalement, la communauté franco-ontarienne se considère comme une communauté culturellement distincte de la communauté ontarienne (anglophone), d'où elle tire d'ailleurs sa légitime revendication des services essentiels adaptés à ses besoins (Office des affaires francophones 1991; ministère de la Culture et des communications 1991; Cazabon et al. 1998). Comment les enseignants de cette même communauté peuvent-ils alors ignorer que la culture de l'apprennant influence aussi ses apprentissages?

\section{L'intégration des minorités visibles $\square$ : au-delà du discours}

«Mais c'est surtout quand est venu le moment d'émettre des suggestions pour aider les étudiants des minorités visibles à contrer les difficultés rencontrées en milieu de stage que ces enseignants identifient leurs camps respectifs. 》
La majorité des 13 enseignants-associés interviewés se disent ouverts et prêts à recevoir des étudiants-maitres des minorités visibles. Cependant, ils appartiennent à deux tendances selon qu'ils sont favorables (4 sur 13) ou non (9 sur 13) à l'intégration des minorités visibles dans la profession enseignante. Mais c'est surtout quand est venu le moment d'émettre des suggestions pour aider les étudiants des minorités visibles à contrer les difficultés rencontrées en milieu de stage que ces enseignants identifient leurs camps respectifs. Les quatre qui sont favorables estiment qu'on devrait favoriser cette intégration pour servir de modèles aux élèves dans les écoles d'une part, et d'autre part, s'ouvrir à d'autres cultures qui représenteraient une richesse pour le patrimoine culturel. Ces quelques enseignants semblent adhérer à l'idée que la diversité culturelle représente un apport indéniable pour la communauté franco-ontarienne 
" "J'ai pris l'attitude de profiter de la présence de cette personne qui a une culture différente de la nôtre pour venir nous enrichir. Je lui ai demandé de parler de son pays et de l'endroit d'où elle venait, d'apporter des choses typiques de son pays "..." (ministère de la Culture et des communications 1991). D'où leur conviction apparente de permettre ou d'élargir l'accès des minorités visibles aux structures, aux ressources et aux circuits déjà existants pour davantage se constituer en une force vive d'une francophonie internationale.

"J'ai pris l'attitude de profiter de la présence de cette personne qui a une culture différente de la nôtre pour venir nous enrichir. Je lui ai demandé de parler de son pays et de l'endroit d'où elle venait, d'apporter des choses typiques de son pays " (EA6, son EM est récent au Canada).

"Ce que je remarque cette année-ci c'est qu'il y en a deux [étudiants-maîtres des minorités visibles],je crois qu'ils sont d'Haiti, alors ils se tiennent un peu ensemble. Je crois que ce n'est pas évident pour eux de devoir aborder un monde comme le nôtre dans l'enseignement parce que quand on regarde le nombre d'enseignants qu'on a dans une école, il y en a une vingtaine et on a une enseignante des minorités visibles. Moi je suis contente de voir qu'il $y$ a d'autres personnes de minorités visibles qui viennent dans les écoles. Pour les enfants ça sera plus rassurant aussi et je crois que c'est comme ça qu'on arrivera à mieux toucher les communautés" (EA12, son EM est récent au Canada).

"Je suis contente d'avoir eu quelqu'un de minorité visible dans ma classe parce que j'ai beaucoup d'élèves haïtiens, somaliens et de peau noire. C'est de bons exemples puis je suis sûre que ce ne doit pas être facile pour eux autres d'être une minorité visible. C'est bon d'avoir quelqu'un de minorité visible qui réussit et qui peut servir de modèles pour les élèves " (EA4, son EM a grandi au Canada).

Toutefois, ces enseignants reconnaissent que cette intégration ne se fera pas toute seule. Entre autres, les conditions de sa réalisation comprennent l'acceptation et le respect de la différence lesquels 
"Plus on est ouvert aux richesses des autres cultures, plus nous autres on y gagne..."

"Il faut commencer à éduquer les profs... qui reçoivent des stagiaires, d'être ouverts au fait que ces gens-là ont droit à un travail, comme n'importe qui d'autres; il faut être ouvert à l'accent, à la couleur." passent par une éducation formelle ou informelle des enseignantsassociés. Curieusement, ces suggestions proviennent des enseignantsassociés ayant supervisé des étudiants-maitres nés à l'extérieur du Canada.

"Ne pas s'attendre à ce qu'ils soient exactement comme nous, je pense que ça serait perdre énormément de la valeur de la diversité des races. Plus on est ouvert aux richesses des autres cultures, plus nous autres on $y$ gagne " (EA6, son EM est récent au Canada).

"Les enseignants-associés se doivent d'être ouverts aux gens, poser des questions, s'intéresser à ce qui se passe. La plupart du temps les gens ne sont pas intéressés à connaître d'autres cultures. S'ils ne sont pas intéressés, ils vont rejeter les autres " (EA10, son EM est récent au Canada).

"Il faut commencer à éduquer les profs [enseignantsassociés] qui reçoivent des stagiaires, d'être ouverts au fait que ces gens-là ont droit à un travail, comme n'importe qui d'autres; il faut être ouvert à l'accent, à la couleur. Il devrait peut-être $y$ avoir des sessions spéciales avant le stage pour ces gens qui vont être mis ensemble " (EA12, son EM est récent au Canada).

Plusieurs auteurs ont déja souligné l'importance d'une formation à la supervision pour les enseignants qui reçoivent des stagiaires dans leur classe (Booth et Hargreaves 1995; Borko et Mayfield 1995; Stephenson 1995). Pour leur part, Bujold et Côté ont mis en évidence les problèmes que rencontrent les enseignants-associés et les stagiaires en cours de stage ainsi que leurs attentes respectives pour un partenariat qui profiterait aux uns et aux autres. Dans notre étude, les enseignants-associés qui appuient l'intégration des minorités visibles dans la profession soulignent que la formation à la supervision devra également comprendre une dimension de sensibilisation aux différences. Ce qui, de façon implicite, constitue un aveu de la non-sensibilité aux différences comme un problème rencontré chez les enseignants-associés de 
"Il ressort clairement de notre recherche que ces enseignants-associés ne reconnaissent qu'une seule culture, "celle de la majorité ", à laquelle tout le monde doit se plier. Il semble toujours $y$ avoir un "nous" qui sépare des " autres ». » la communauté franco-ontarienne, tel que l'illustrent d'ailleurs les lignes qui suivent.

Neuf des 13 enseignants-associés apparemment défavorables à l'intégration des minorités visibles dans la profession enseignante semblent nier toute responsabilité dans le sort réservé à ces dernières. C'est pourquoi ils préconisent des mesures visant à aider les minorités visibles à surmonter les obstacles tout en affirmant qu'ils attachent de l'importance à la liberté et à l'égalité des chances.

"Je crois que c'est important qu'on traite les gens également, qu'on ne fasse pas des outils et dire : vous autres vous êtes spéciaux, différents. S'il y a un outil qui est valable en soi, il est valable pour tout le monde; si on a des améliorations à apporter, ça serait pour tout le monde, pas simplement pour les minorités visibles » (EA6, son EM est récent au Canada).

Il ressort clairement de notre recherche que ces enseignantsassociés ne reconnaissent qu'une seule culture, « celle de la majorité ", à laquelle tout le monde doit se plier. Il semble toujours y avoir un « nous » qui sépare des " autres ». Et le souci de protéger cette culture semble si grand qu'il pousse certains des enseignants-associés à faire des suggestions qui ne peuvent être d'aucun apport pédagogique, tout au moins pour les étudiants-maitres, tel que le démontrent les propos suivants :

"Si quelqu'un vient d'un autre pays pour son stage, il faudrait le conscientiser à la population parce qu'il ne connaît pas notre communauté. Il faut lui montrer comment vivent les gens au Canada. Il doit se plier à la nouvelle société " (EA1, son EM est né au Canada).

"Il faudrait des activités qui pourraient faire vivre la culture francophone aux étudiants-maîtres des minorités visibles: sorties éducatives, implication au niveau culturel, journée dans la ville de Québec, aller observer les gens dans les centres d'achats, examiner leurs comportements" (EA9, son EM est né au Canada). 
"Quand c'est un chinois qui parle, ce n'est pas facile. Moi, j'ai accouché avec une chinoise comme infirmière, puis je vous le jure je n'ai pas apprécié. Je m'excuse c'est une digression mais ça revient au même! »
"On est dans une profession où on doit s'exprimer en public, de façon à être compris par tout le monde. Alors tu sais c'est très important, ils doivent travailler davantage la phonétique pour que les élèves comprennent mieux l'élocution. Ce serait l'observation la plus urgente, je pense, et c'est faisable. Vous voyez des "speakerines" à Radio-Canada qui sont d'origine haïtienne et qui s'expriment merveilleusement bien; on les comprend tous. Alors les enseignants devraient être pareils. Si vous avez un enseignant de chimie qui est d'une autre identité, d'une autre ethnie, ce n'est pas facile! Les formules chimiques vous les entendez moins :il ne faut pas avoir des problèmes d'ouïe, il faut essayer de se casser le cou pour être le premier en avant de la salle de classe, parce qu'on a du mal à entendre! C'est parce qu'eux, l'accent tonique n'est pas au même endroit que nous... Quelque chose pour les aider à ce niveau, c'est d'offrir des cours d'élocution qui sont d'un français international au moins, donner des cours de phonétique même! Est-ce que vous êtes allé [s'adressant à l'intervieweur] à l'école, à l'université avec un professeur d'une autre ethnie? Quand c'est un chinois qui parle, ce n'est pas facile. Moi,j'ai accouché avec une chinoise comme infirmière, puis je vous le jure je n'ai pas apprécié. Je m'excuse c'est une digression mais ça revient au même! " (EA8, son EM est né au Canada).

N'est-il pas regrettable de constater que ces enseignants-associés, qui s'opposent catégoriquement à l'intégration des minorités visibles en proposant des solutions enfantines ou à tout le moins dégradantes, ont supervisé des étudiants-maîtres nés au Canada et formés par ce même système qui les rejette aujourd'hui? Ceux des étudiants-maitres de minorités visibles qui ne sont pas victimes du nom qu'ils portent sont victimes de la couleur de leur peau ou de leur accent ou plutôt de leur langue comme le dit tout haut cet enseignant-associé : 
"Ils ont le vocabulaire précis mais personne [ne] les comprend, donc ils ne peuvent pas enseigner à nos jeunes, ne peuvent pas fonctionner dans nos écoles"..."
"La langue, c'est très très important : la prononciation des mots pour que les élèves les comprennent. Ils ont le vocabulaire précis mais personne [ne] les comprend, donc ils ne peuvent pas enseigner à nos jeunes, ne peuvent pas fonctionner dans nos écoles " (EA1, son EM est né au Canada).

Les dires de ces participants nous ramènent aux propos de Jodelet : "Qu'est-ce qui fait que, dans des sociétés qui se réclament de valeurs démocratiques et égalitaires, les gens soient amenés à accepter l'injustice, à adopter ou à tolérer, vis-à-vis de ceux qui ne sont pas des leurs ou comme eux, des pratiques de discrimination qui les excluent?» $(1996: 67)$.

\section{Conclusion}

"..."l'effet des vécus minorisés, des appartenances fragilisées se manifeste chez les enseignants et les cadres ". "
Afin de préserver l'hégémonie de la communauté franco-ontarienne de "souche », on constate, à la lumière de leurs témoignages, que la majorité des enseignants-associés semblent réticents à ce que les minorités visibles intègrent la profession enseignante. Cette étude nous aura permis de dépeindre le comportement d'intervenants de diverses institutions d'enseignement, confrontés au défi du pluralisme ethnoculturel. Elle constitue par ce fait même un premier développement à partir duquel on peut mieux réfléchir à la nécessité de revoir les programmes de formation des maitres, les stages et particulièrement la formation des enseignants-associés en Ontario français. Cazabon et al. (1998 : XI) en arrivent quasiment au même constat : «l'effet des vécus minorisés, des appartenances fragilisées se manifeste chez les enseignants et les cadres ». Ces auteurs s'interrogent sur le genre de travail de formation initiale et continue qui doit être entrepris «pour modifier les perceptions qui contredisent la possibilité même d'entreprendre un changement ". À ce propos, des suggestions émises par certains enseignants-associés ayant participé à notre étude semblent des voies pertinentes qui pourraient être valables pour l'ensemble des acteurs : des sessions et des ateliers 
"D'après leurs témoignages, la majorité des enseignants-associés participant à cette étude adhèrent, selon nous, au modèle traditionnel. "
"Cette critique semble un peu ethnocentrique compte tenu de la qualité de la langue des étudiants francoontariens en général...» de formation et de sensibilisation pour préparer les membres de la majorité à recevoir, encadrer et accepter les étudiants-maîtres et les futurs enseignants des minorités visibles et, par le même biais, les éduquer à être ouverts à l'accent et à la couleur.

À cette fin, Anderson (1992) distingue deux modèles de supervision des stages : un traditionnel et un clinique. Selon elle, le modèle traditionnel est orienté vers les activités propres à un processus de sélection soit, l'évaluation et la certification des candidats; le modèle clinique est orienté vers la formation des stagiaires, par l'application de démarches de résolution de problèmes et par l'établissement d'une relation d'aide. D'après leurs témoignages, la majorité des enseignants-associés participant à cette étude adhèrent, selon nous, au modèle traditionnel. Pour les préparer plus adéquatement à leurs rôles de facilitateur, de mentor, d'aidant ou de parrain, la formation devra s'inscrire dans une perspective de supervision clinique.Vantant les mérites de la supervision clinique, Coe (1985) soutient qu'elle suppose une supervision chaleureuse mettant l'accent sur l'amélioration de l'intervention en classe. Elle offre une occasion non seulement d'effectuer un retour sur soi, mais aussi de s'améliorer et d'améliorer sa pratique professionnelle.

Dans un contexte franco-ontarien, fournir aux enseignantsassociés une occasion de retour sur soi nous semble une suggestion des plus opportunes, surtout face à la question de la langue, de l'accent et de la tonalité de la voix des étudiants-maitres que ces mêmes enseignants identifient comme le noeud des problèmes rencontrés chez les minorités visibles en stage. Selon ces enseignants-associés, l'accent des étudiants-maitres des minorités pose un problème sérieux pour les élèves qui ont beaucoup de difficultés à comprendre les interventions pédagogiques de ces derniers qui, par ailleurs, sembleraient être plutôt faibles en français. Cette critique semble un peu ethnocentrique compte tenu de la qualité de la langue des étudiants franco-ontariens en général (Cazabon et al. 1998). Un retour sur soi permettrait donc à ces enseignants-associés de se découvrir et de réaliser que, par rapport à leurs nombreux élèves membres des minorités visibles (dont les proportions augmentent de plus en plus) et aux stagiaires des 
"Dès lors, l'accent ne serait plus un mal dont les minorités visibles doivent se guérir."

"L'accent d'un individu n'est pas quelque chose qu'on peut réparer. C'est une partie intégrante de l'individu qui le distingue d'un autre et qui est révélateur de son appartenance à une communauté linguistique ou culturelle donnée." minorités visibles, ils ont eux-mêmes un accent. Ils pourraient ainsi prendre conscience du fait que l'effort qu'ils investissent à comprendre les minorités visibles est le même que ces dernières investissent pour comprendre les Franco-Ontariens de souche et pour s'adapter aux nuances régionales franco-ontariennes. Dès lors, l'accent ne serait plus un mal dont les minorités visibles doivent se guérir.

En effet, un enseignant-associé, invité à nous faire part de ses suggestions pour améliorer la condition des étudiants-maitres de minorités visibles, a recommandé d'envoyer ces étudiants suivre "des cours d'élocution qui sont d'un français international... des cours de phonétique ». Ce concept de phonétique internationale nous semble impensable et ne fait que renforcer le manque de sensibilité aux différences évoqué plus haut. L'accent d'un individu n'est pas quelque chose qu'on peut réparer. C'est une partie intégrante de l'individu qui le distingue d'un autre et qui est révélateur de son appartenance à une communauté linguistique ou culturelle donnée. L'accent fait également partie de l'hétérogénéité linguistique qui elle-même est caractéristique de la diversité culturelle qui devient, de nos jours, une réalité sociale qu'il faudrait plutôt accueillir et faire fructifier pour le bien de toute la communauté.

En tant que milieux d'apprentissage et de formation, les facultés d'éducation et les établissements scolaires qui reçoivent des stagiaires devraient s'ouvrir au concept de l'adaptation et accepter que celle-ci se fasse dans les deux sens. Comment y arriver? Un profond questionnement de notre propre système de valeurs s'impose face à celui des autres afin de rendre les frontières plus perméables. Par exemple, il ne suffirait pas d'encourager les étudiants des minorités visibles à suivre des cours sur la culture franco-ontarienne, mais il faudrait aussi encourager l'ouverture de cette dernière communauté à la réalité culturelle de ces étudiants. Il ne s'agirait pas d'envoyer ces étudiants faire de l'observation dans les centres d'achat dans la ville de Québec pour voir comment les gens vivent au Canada, mais plutôt de leur permettre de s'intégrer dans les groupes informels afin qu'ils aient accès aux codes culturels franco-ontariens. Il ne faudrait pas 
"Des études plus approfondies devraient permettre de tenir compte des représentations dont sont porteurs les divers partenaires des milieux universitaire et scolaire directement impliqués dans la formation des enseignants, sur l'intégration des minorités visibles et ethnoculturelles dans la profession enseignante. " organiser des journées multiculturelles pour eux, afin de leur donner l'opportunité de se regrouper, mais plutôt organiser des journées multiculturelles axées sur la diversité. Enfin, il ne suffit pas de dire qu'ils ne sont pas qualifiés pour enseigner à nos jeunes, il faut trouver des façons de s'assurer qu'ils le soient. Une responsabilité à assumer, diraient certaines gens. Mais à qui revient-elle?

Ce rôle revient principalement aux institutions de formation des enseignants, à savoir les facultés d'éducation et les écolesassociées. Dans notre étude, nous nous sommes limitée aux perceptions des enseignants-associés. Des études plus approfondies devraient permettre de tenir compte des représentations dont sont porteurs les divers partenaires des milieux universitaire et scolaire directement impliqués dans la formation des enseignants, sur l'intégration des minorités visibles et ethnoculturelles dans la profession enseignante. Il reste également à mener d'autres études en comparaison avec des étudiants-maitres des minorités visibles des milieux francophones majoritaires et anglophones pour mettre en lumière la part qui revient au facteur "francophone minoritaire ». Finalement, il importe d'explorer des moyens visant à accroître le nombre des représentants des minorités visibles et ethnoculturelles et à faciliter leur insertion effective dans la profession enseignante. Au-delà de la profession enseignante, la démarche que nous proposons peut offrir des pistes aux études ultérieures sur la condition des minorités visibles dans d'autres domaines de l'activité humaine qui restent, pour la plupart, inexplorés.

\section{Bibliographie}

ABELLA, R.S. (1984). Rapport de la commission sur l'égalité en matière d'emploi. Gouvernement du Canada.

ALLADIN, M.I. (1996). Racism in Canadian Schools. Toronto, Harcourt Brace.

ANDERSON, D.J. (1992). A Quantitative Analysis of Student Teacher Supervision Models : Implications for the Role of University Supervisors. Document ERIC ED353252.

APPlEGATE, J.H. et LESLEY,T. J. (1982). « Cooperating Teachers' Problems with Preservice Field Experience Students ", Journal of Teacher Education, 33 (2), 15-18.

AXELROD, P. (1997). The Promise of Schooling. Toronto, University of Toronto. 
BARTON, W., WATKINS, H.M, et JARJOURA, R. (1997). "Youths and Communities : Toward Comprehensive Strategies for Youth Development ", Social Work, 42 (5), 483-493.

BECKHAM, B. (1988). «Stranger in a Strange Land :The Experience of Blacks on White Campuses », Educational Record, 69 (1), 74-78.

BIRCH, D.R. et ELLIOT, M. (1993). "Towards a new Conception of Teacher Education ", in L. Stewin et S. McCann (Eds.), Contemporary Canadian Issues, The Canadian Mosaic, Toronto, Copp Clark Pitman Ltd, 353-379.

BIRON, D. et al. (1995). "Sur la formation à la pratique en enseignement des mathématiques au primaire ", Bulletin, AMQ, vol. XXXV, no 1, mars 1995, 50-52.

BOISVERST, S. (1987). Devrait-on donner une formation aux maitres-associés des stages des programmes de formation des maîtres? Comparaison de la situation actuelle et de la situation anticipée. Mémoire de maitrise, Montréal, Université du Québec à Montréal.

BOOTH, M. et HARGREAVES, D.H. (1995). «Training Doctors in Hospitals : A Comparison with Teacher Education ", Journal of Education for Teaching, 21 (2), 145-161.

BORKO, H. et MAYFIELD, V. (1995). "The Roles of the Cooperating Teacher and University Supervisor in Learning to Teach ", Teaching and Teacher Education, 11(5), 501-518.

BUJOLD, N. et CÔTÉ, É. (1996). «Étude préalable à l'implantation d'un programme de formation pour la supervision des stages en enseignement ", Revue des sciences de l'éducation, vol. XXII, no 1, 25-46.

CARR, P.R.(1995). "Employment Equity for Racial Minorities in the Teaching Profession ", Multicultural Educational Journal, 13 (1), 28-42.

CAZABON, B. et al. (1998). "La pédagogie du français langue maternelle et l'hétérogénéité linguistique ", Québec, Centre international de recherche en aménagement linguistique international.

CHENG, M. et SOUDACK, A. (1994). Antiracist Education : A Literature Review. Toronto, Toronto Board of Education.

CLAY,J, GADHIA, S., et WILKINS, C. (1991). « Racism and Institutional Inertia : A 3-D Perspective of Initial Teacher Education (Disillusionment, Disaffection and Despair) », Multicultural Teaching, 9(3), 26-31.

COE, D, E. (1985). Toward Collegial Inquiry : A Case Study in Clinical Supervision, Document ERIC ED281847.

DEI, G.J.S. et al. (1997). Reconstructing Drop-out : A Critical Ethnography of the Dynamics of Black Students' Disengagement from School. Toronto, UTP.

DEI, G.J.S. (1996). Anti-Racism Education Theory and Practice. Halifax, Fernwood Publishing.

DEI, G.J.S. (1993). "The Challenges of Anti-racist Education in Canada », Canadian Ethnic Studies, XXV, 36-51.

DESCÔTEAUX, J. M. (1992). «Le stage en milieu professionnel dans la formation en tourisme », TÉOROS, vol.11, no 1, mars 1992,17-23.

FRIESEN, J.W. (1993). «Implications of Ethnicity for Teaching », in L. Stewin et S. McCann (Eds.), Contemporary Canadian Issues, The Canadian Mosaic, Toronto, Copp Clark Pitman Ltd., 89-96.

GIOR GI,A. (1985). Phenomenology and Psychological Research. Pittsburgh, Duquesne Press.

GOLLNICK, D.M. et Chinn, P.C. (1998). Multicultural Education in a Pluralistic Society. 5th ed. N.J., Merill. 
GOODWIN, C.D. et M. Nacht. (1983). Absence of Decision. New-York, Institute of International Education.

GRANT, C. (1993). «The Multicultural Preparation of U.S.Teachers : Some HardTruths », in G.Verma (Ed.), Inequality and Teacher Education, London, Falmer Press, 41-57.

HENRY, F. et al. (2000). The Colour of Democracy : Racism in Canadian Society $2^{\text {nd }}$ ed.). Toronto, Harcourt Brace \& Company Canada.

HENRY, F. et al. (1995). The Colour of Democracy : Racism in Canadian Society. Toronto, Harcourt Brace.

HENRY, F. etTATOR, C. (1994). "The Ideology of Racism-Democratic Racism », Canadian Ethnic Studies, 26 (2), 1-14.

HERRY,Y. (1995). Programme de formation initiale en langue française adapté aux besoins des membres des minorités raciales et des autochtones de l'Ontario. Document non publié.

HUNTLEY, H.S. (1993). Adult International Students : Problems of Adjustment. ED355886.

JODELET,D. (1996). « Les processus psycho-sociaux de l'exclusion ", dans S. Paugam (Ed.), L'exclusion, l'état des savoirs, Paris, La Découverte, 66-77.

LANGLOIS, H. (1998). L'école franco-ontarienne à l'heure de la diversité culturelle : Outil pédagogique pour une éducation inclusive. Ottawa, CFORP.

LAVOIE, M. et GARANT, C. (1995). «Les stages d'enseignement comme lieu privilégié de partenariat école et université ", Bulletin AMQ, vol XXXV, no 1, mars 1995, 32-48.

L'ÉCUYER, R. (1987). «L'analyse de contenu : notion et étapes », dans Deslauriers, J.-P. Les méthodes de la recherche qualitative. Québec, Presses de l'Université du Québec.

LEGENDRE, R. (1988). Dictionnaire actuel de l'éducation. Québec, Université du Québec à Montréal.

LOCKE, L.F. (1984). " Research on Teaching Teachers :Where Are We Now? ", Journal of Teaching in Physical Education, Summer Monograph, 2, 3-86.

MARTIN,Y.M. et WARBURTON, R. (1998). Voices for Change : Racism, Ethnocentrism and Cultural Insensitivity at the University of Victoria. A report submitted to David Strong, President, University of Victoria.

MCCONNELL Castle, E. (1993). «Auditing African-American and Hispanic Student Attrition and Satisfaction in University Environments : Can We Learn to Retain Them? ", in People and Education, 1(4), 393-413.

MINISTÈRE DE LA CULTURE ET DES COMMUNICATIONS (1991). Rêver, sentir, vouloir, pouvoir (RSVP!). Toronto, L'imprimeur de la Reine pour l'Ontario.

MINISTÈRE DE L'ÉDUCATION ET DE LA FORMATION DE L'ONTARIO (1994). Demandes de propositions dans le cadre des initiatives d'antiracisme reliées à la formation du personnel enseignant. Toronto : Ministère de l'éducation et de la formation de l'Ontario.

MUJAWAMARIYA, D. (sous presse). Les minorités visibles face à la pratique d'enseignement : leçons à tirer d'expériences d'étudiants-maitres dans un contexte francophone minoritaire. Actes de Colloque de l'AQUFOM.

MUJAWAMARIYA, D. (1998). "Should “Where Are You From” Sound Good? ", Summer English Language Program Journal, University of Victoria, 15.

NIXON-PONDER, S. (1998). "Teens and Schools : Who is Falling Through the Cracks and Why », in J.S. Epstein (ed.), Youth Culture : Identity in a Postmodern World, Malden, Ma, Blackwell Publishers, 56-73. 
OFFICE DES AFFAIRES FRANCOPHONES (1991). Rapport annuel 1990-1991. Toronto, Office des affaires francophones.

OUELLET, F. (1999). «L'éducation face aux défis du pluralisme ethnoculturel. Les grandes questions de l'heure ", Association pour la recherche interculturelle, Bulletin no 32, France, Université Paris XNanterre, 9-19.

PARÉ, A. (1993). « Présence en éducation », Intégration, 17, 9-33.

SATZEWICH,V.(Ed.) (1998). Racism \& Social Inequality in Canada : Concepts, Controversies \& Strategies of Resistance. Toronto, Thompson Educational Publishing, Inc.

SLEETER, C.E. (1993). "How White Teachers Construct Race ", in C. McCarthy et W. Crichlow (Eds.), Race Identity and Representation in Education, New York and London, Routledge, 157-171.

SMITH, L. (1989). Perspectives on Teacher Supply and Demand in Ontario, 1988-2008. Toronto, Queen's Printer for Ontario.

SOLOMON, R.P. (1999). "The Challenges of Pre-Service Teachers of Colour in Negociating Equitable Space in Practica ", Summary of Paper Presented at The Congress of the Social Sciences and Humanities. Université de Sherbrooke.

SOLOMON, R.P. (1997). "Race, Role-modelling and Representation in Teacher Education and Teaching ", Canadian Journal of Education, 22 (4), 395-410.

SOLOMON, R.P. (1995). "Why to Teach from a Multicultural and Antiracist Perspective in Canada. Race, Gender and Class ", An Interdisciplinary and Multicultural Journal, 2 (3), 49-66.

SPALLANZANI, C. et al. (1992). «Effets d'une formation minimale à la supervision de stagiaires en éducation physique ", Revue des sciences de l'éducation, XVIII (3), 409-427.

STANLEY, T.J. (1995). "White Supremacy and the Rhetoric of Educational Indoctrination : A Canadian Case Study ", in J. Barman, N. Sutherland et J.D. Wilson (Eds), Children, Teachers and Schools in the History of British Columbia, Calgary, Delselig Enterprises, 39-56.

STATISTIQUE CANADA (1998). Population des minorités visibles : recencement de 1996. Ottawa, Statistique Canada.

STEPHENSON, J. (1995). «Significant Others :The Primary StudentView of Practice in Schools ", Educational Studies, 21 (3), 323-335.

TALBERT-JOHNSON, C. et TILLMAN, B. (1999). «Perspectives on Colour in Teacher Education Programs : Prominent Issues ", Journal of Teacher Education, 50, 200-208.

VARPALOTAI, A. (1995). "Affirmative Action for a Just and Equitable Society ", in R. Ghosh \& D.Ray (Eds.), Social Change and Education in Canada, 3rd edition, Toronto, Harcourt Brace, 240253.

VILLEMURE, M. (1989). La formation à l'enseignement : sortir de l'impasse? Suivi de trois esquisses : les qualités du bon professeur, le temps présent, la culture générale. Ottawa, Les Éditions du Vernillon.

WADE, M. (1974). "Commentaire " Actes de colloque - Situation de la recherche sur la vie française en Ontario, Ottawa, CRCCF et ACFAS, 79-82.

WATT, D. \& Roessingh, H. (1994). Inclusion and Language Minority Education : Curricular Implications. Faculty of Education, University of Calgary.

WIDEEN, M., MAYER-SMITH, J. and MOON, B. (1998). "A Critical Analysis of Research on Learning to Teach : Making the Case for an Ecological Perspective on Inquiry ", Review of Educational Research, 68 (2), 130-178. 
YOUNG, G.L. (1994). Looking Through Eyes of Compassion :Visible Minorities in SIAST. Saskatoon, YLB.

YOUNG, I.M. (1990). Justice and Politics of Difference. New-Jersey, Princeton University Press.

ZEICHNER, K. \& Hoeft, K. (1996). « Teacher Socialization for Cultural Diversity », in Handbook of Research on Teacher Education, New York : Macmillan, 525-547.

ZEICHNER, K. (1980). "Myths and Realities : Field-based Experiences in Preservice Teacher Education ", Journal of Teacher Education, 31 (6), 45-55.

\section{Notes}

1. L'expression étudiant-maitre désigne l'étudiant en formation qui se prépare à devenir enseignant. Quant à lui,l'enseignant du primaire ou du secondaire qui supervise cet étudiant en situation de stage pratique à l'enseignement est appelé enseignant-associé.

2. Appartiennent à cette catégorie les Canadiens qui estiment avoir, entièrement ou partiellement, une origine non européenne et non autochtone et qui sont visiblement reconnaissables comme tels. Ordinairement, il s'agit de personnes dont les ancêtres étaient originaires d'Asie, d'Afrique, d'Amérique du Sud, D'Amérique Centrale et des Îles du Pacifique.

3. L'auteure adresse ses sincères remerciements au ministère du Patrimoine canadien et à la Faculté d'éducation de l'Université d'Ottawa pour avoir financé cette étude. Ces remerciements s'adressent également à Denyse Pharand, Dominic Ambroise, Sylvie Beaulne, à tous les participants et toutes les participantes pour leur précieuse collaboration dans la réalisation de cette étude.

4. Un groupe ethnoculturel se compose de personnes qui possèdent en commun le même patrimoine culturel ou les mêmes antécédents culturels. Il existe plusieurs groupes ethnoculturels parmi les Africains, les Asiatiques et les Européens et parmi les Autochtones de l'Amérique du Nord, de l'Amérique Centrale, de l'Amérique du Sud et de l'Australie qui vivent au Canada.

5. Peuples formés des descendants des premiers habitants du Canada dont les Indiens, les Inuits et les Métis.

6. Dans cet article, nous nous limiterons à présenter les résultats de notre étude auprès des enseignantsassociés. Les données recueillies auprès des étudiants-maitres de minorités visibles feront l'objet de publications à venir. 